\title{
An image processing-based system proposal for real-time detection of drowsiness from a vehicle driver's eye movements
}

\author{
${ }^{* 1}$ Murat Silahtaroğlu and ${ }^{2}$ Serkan Dereli \\ ${ }^{* 1}$ Department of Electrical and Electronics Engineering, Sakarya University of Applied Sciences, Turkey \\ ${ }^{2}$ Department of Computer Technologies and Programming, Sakarya University of Applied Sciences, Türkiye
}

\begin{abstract}
Drowsiness is one of the underlying causes of driving accidents leading to serious injuries and deaths annually. According to the experts has mentioned that almost $30 \%$ of all traffic accidents have been caused by drowsiness. In avoiding these traffic accidents, a proper system is required to prevent the driver from falling asleep. This study proposes a real-time image processing-based system for recognizing the drowsiness face expression of the vehicle driver. The method of this study, detecting the exact position facial landmarks and both left and right eyes using dlib and eye aspect ratio algorithm. This system, after detecting drowsiness eye, give audible alert the vehicle driver to stay awake throughout the driving journey.
\end{abstract}

Key words: Eye detection, image processing, drowsiness, real-time detection

\section{Introduction}

The increasing number of traffic accidents due to a driver's diminished vigilance level has become a serious problem for society. Some of these accidents are the result of the driver's medical condition. However, a majority of these accidents are related to driver fatigue, drowsiness of drivers. Car accidents associated with driver fatigue are more likely to be serious, leading to serious injuries and deaths [1]. Detecting the levels of drivers' drowsiness has a key role in reducing the number of fatal injuries in traffic accident. Recent statistics and reports show that 20 to 50 million people are killed or injured in car crashes all over the world [2].

Detecting driver drowsiness systems can be classified into two categories: contact types and contactless types. Contact types include measuring the pulse or body temperature, while detecting and analyzing the driver's facial expression is a standard contactless type [3]. However, the products requiring physical contact are inconvenient for drivers and easily forgotten. On the other hand, most commercially available contactless driver drowsiness detection systems and related products use visible light to achieve face detection [4]. During the day, they do not affect drivers; nevertheless, at night, since these systems need to gather the skin-color region, they are likely to increase the burden on driver's eyes since extra light was projected on the driver's face to properly obtain color images [5].

*Corresponding author: Murat Silahtaroğlu, Address: Department of Electrical and Electronics Engineering, Sakarya University of Applied Sciences, Turkey, 54187, Sakarya TURKEY. E-mail address: muratsilahtaroglu13@gmail.com, Phone: +902646160492 
Currently, there are studies for the creation of a drowsiness detection system, which extracts the essential characteristics of drowsiness of the driver to determine the level of drowsiness. In these systems, which are controlling the state of drowsiness of the driver through a webcam with night vision to track the driver in real time and when drowsiness is detected, the system will send a warning [6]. The objective of these systems is to improve the safety of people with the use of detection and alarm to avoid accidents caused by drowsiness of the driver and which are harmful to the users of the route [7].

The aim of this study focuses on building a real-time model, using image based information and a template of eye region in human face. If driver closes eye/s for some particular time then system will generate the alarm. It is non-intrusive system for monitoring driver drowsiness based on open and close conditions of eyes. Eye behaviors provide significant information about driver's alertness and that if visual behavior can be measured then it will be feasible to predict driver's state of drowsiness, vigilance or attentiveness.

\section{Materials and Method System Structure}

The system features three main parts as shown figure 1. First, the system collects the necessary data to locate the driver's face region and eyes; second, the system analyzes the captured data and collects new data from the images to set the fatigue model. Once the model is built, the system then starts to detect the driver's physical state. If the eye size level is smaller than the predetermined eye size threshold, system understand that there is drowsiness. Finally, if the driver shows signs of drowsiness, an alarm sounds to warn the driver.

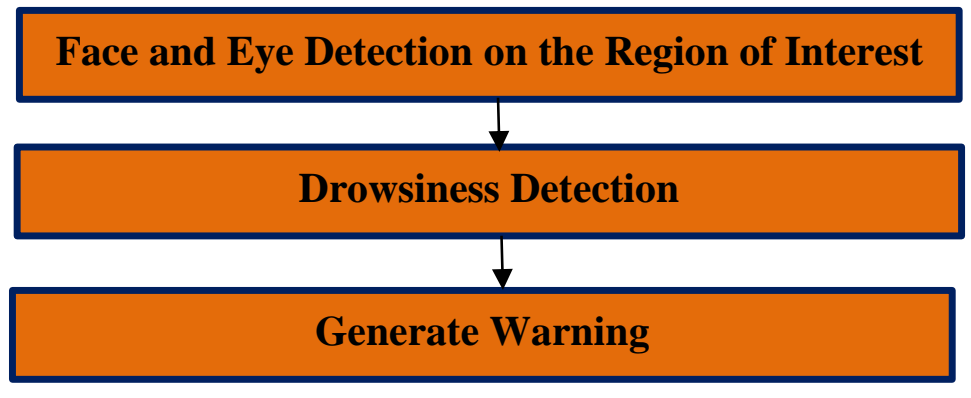

Figure 1: Block diagram of the system structure

\subsection{Face and Eye Detection}

In this project, it was detected and extracted facial landmarks using dlib, OpenCV, and Python. Detecting facial landmarks is a two step process:

The first step process, localizing the face in the image. Real-time face recognition shown figure 2 , this process is face detection, which is one of the numerous object identification applications. Because of human diversity the precise recognition of human faces is much more hard process then it expected. It was used OpenCV's built-in Haar cascades. 

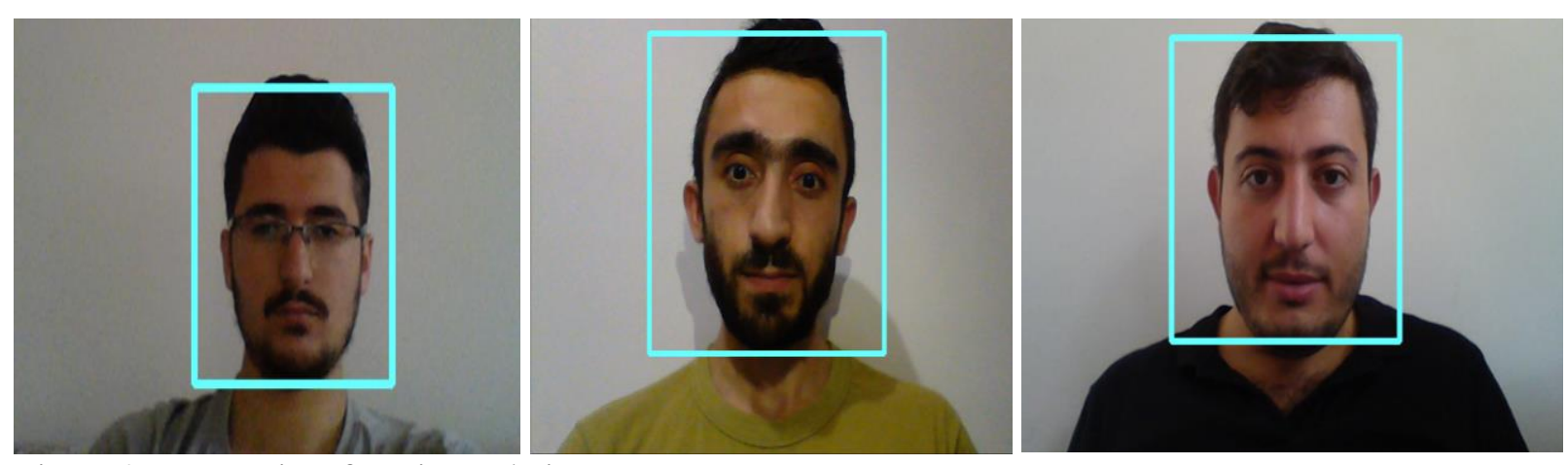

Figure2: Detecting face in real-time

The second step process, detecting important facial structures on the face Region of Interest. In this stage, it uses image processing to detect anterior faces with facial landmark detection of mouth, nose, right and left eyebrow, right and left eye, and jaw. It was implemented facial landmark detection by using dlib library.

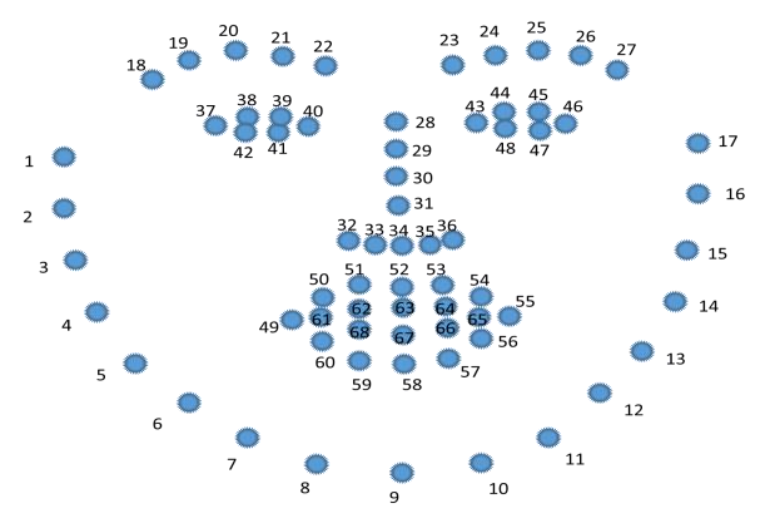

Figure 3: Facial landmark coordinates

In the dlib library, the pre-trained facial landmark system is used to predict the location of 68 coordinates on the face that matches the facial structures.

The indices of 68 coordinates are shown in figure 3.

It was detected eyes recognition from facial landmark system which is certain right and left eye coordinates. Right and left eye is expressed by $6(\mathrm{x}, \mathrm{y})$-coordinates as shown figure 4 .

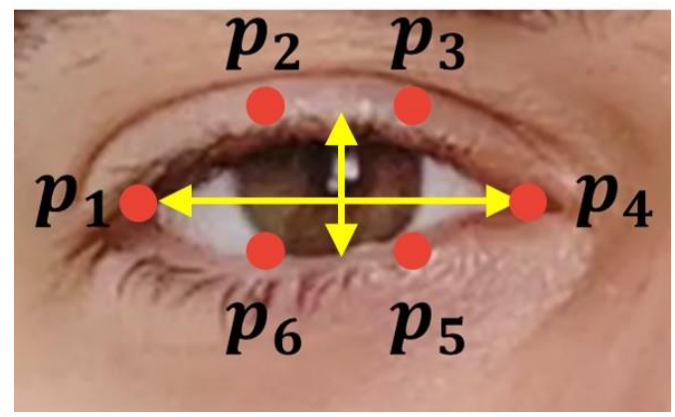


Figure 4: The 6 facial landmarks related with the eye.

After eye recognition was implemented, it was drawn eye shape around eyes as shown figure 5 in real-time.
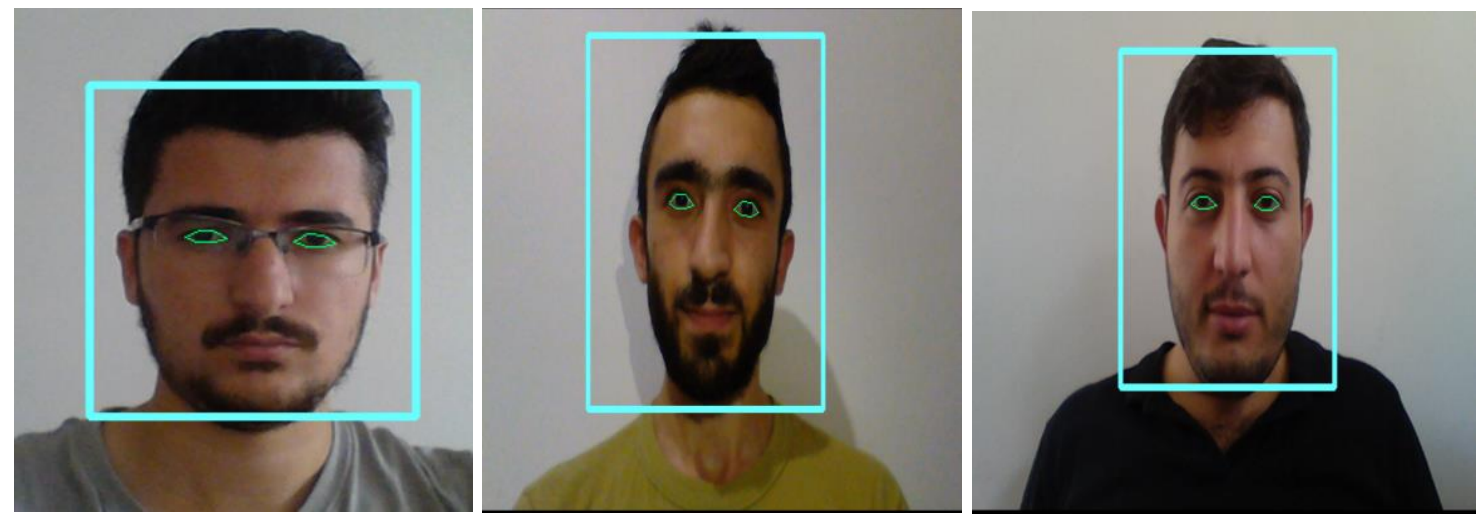

Figure 5: Detecting eye in real-time

\subsection{Drowsiness Detection and Generate Warning}

There is a relation between the width and the height of eyes location which have 6 (x, y) coordinates. To detect drowsiness, it was used that this relation called the eye aspect ratio (EAR) shown figure 6.

$$
\mathrm{EAR}=\frac{\left\|p_{2}-p_{6}\right\|+\left\|p_{3}-p_{5}\right\|}{2\left\|p_{1}-p_{4}\right\|}
$$

Figure 6: The eye aspect ratio formula

The numerator of this formula computes the distance between the vertical eye landmarks while the denominator computes the distance between horizontal eye landmarks, weighting the denominator appropriately since there is only one set of horizontal points but two sets of vertical points in show figure $6[8]$.
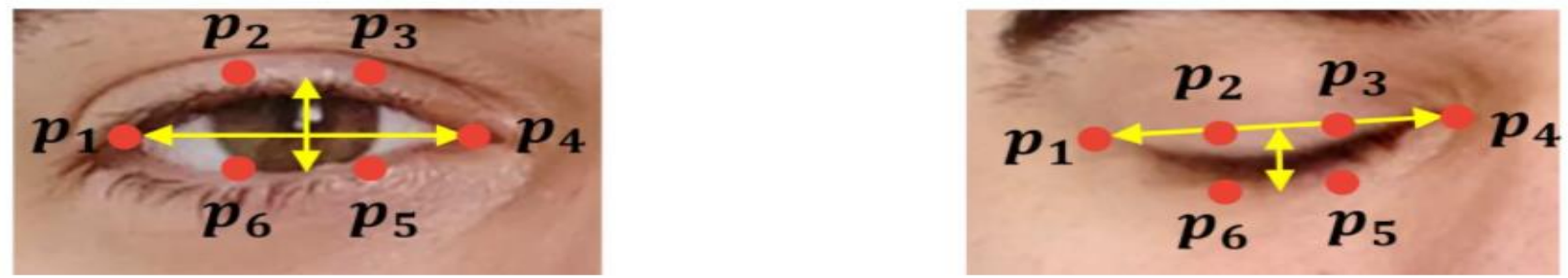

Figure 7: Top-left: A visualization of eye landmarks eye is open. Top-right: Eye landmarks when the eye is closed.

Using in the figure 6 basic formula, it was refrain from image processing techniques and used the 
eye aspect ratio determine for a person is drowsiness. It is easily understandable that eye openness level or closed eyes as shown figure 7. The eye size level is smaller than the predetermined eye size threshold, system understand that there is drowsiness. After detecting drowsiness driver in real-time, alarm sounds to warn as in show figure 8.
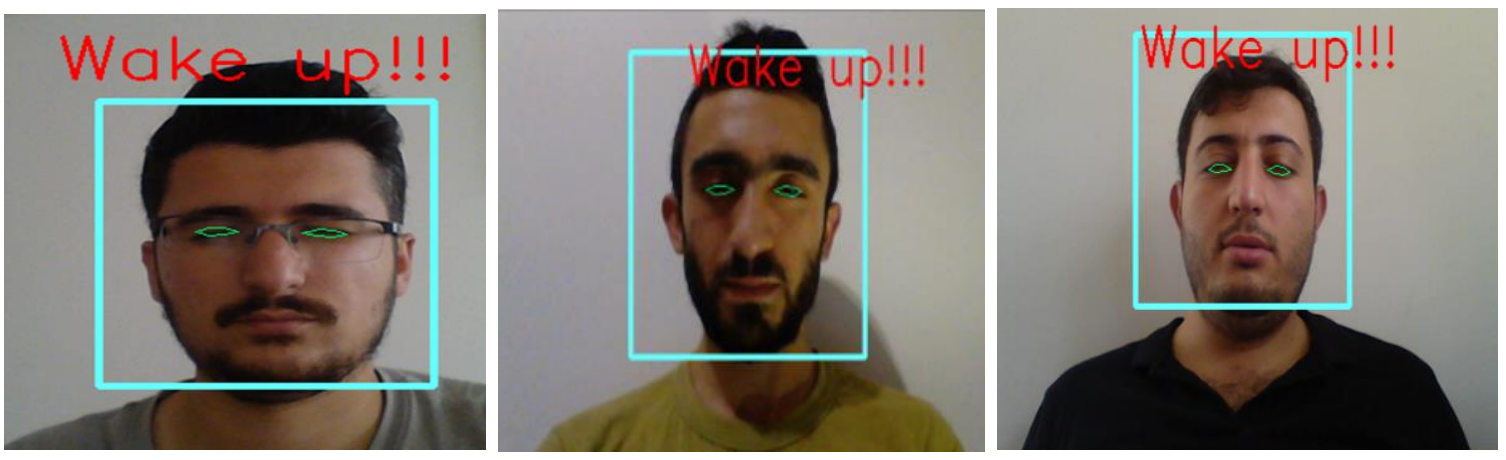

Figure 8: Detecting drowsiness in real-time and generate warning

\section{Result}

The tests were performed on 3 people with different eye sizes in real time. And the average success rate is $88.2 \%$. It is $95.4 \%$ in the tests made with the dataset consisting of approximately 1000 photographs consisting of open, half-open and closed eyes. In table 1, it was shown the recognition precision obtained from four approaches results which consisting of average of standard datasets and real-time.

The difference of this study from other studies is that it supports both our own data set and different data sets. When the first time the system works, the eye size of the person is measured as real-time. After that, the eye aspect ratio is calculated and the threshold value is determined. Thus, it has a dynamic working structure independent of the person's eye size.

\begin{tabular}{|c|c|c|}
\hline \multicolumn{3}{|c|}{ Recognition Precision } \\
\hline Research & Methodology/Algorithms & Accuracy \\
\hline$[9]$ & Blinking feature + EOG & $\% 82.1$ \\
\hline$[10]$ & Boost-LBP + SVM & $\% 85.9$ \\
\hline
\end{tabular}




\begin{tabular}{|c|c|c|}
\hline$[11]$ & Gabor + SVM & $\% 91.45$ \\
\hline Ours & Blinking + Dlib + EAR & $\% 92.1$ \\
\hline
\end{tabular}

Table 1: Recognition precision results

\section{Conclusions}

In this study, first the face was detected, and then the eye was detected from facial landmarks features. An image processing-based system was developed by taking the eye as a reference in order to detect the drowsiness status of the drivers in the vehicle. All results show that image processing method an efficient way for drivers' drowsiness detection. Regardless of the size of the eye, drowsiness was accurately detected. Therefore, accidents caused by drowsiness will be mostly avoided. The most important feature emphasized in this recommendation is the detecting of beginning of the moment of drowsiness. Thus, regardless of the eye size of the person, it contributes to a safer drive thanks to early warning. To obtain more reliable results, it will be added yawning detected from facial landmarks features.

This study has structure that it can be integrated on the embedded system and can be converted into a portable form. Thanks to this, it will be installed in any vehicle with a suitable microprocessor and it will be provided to use actively.

\section{References}

[1] Fletcher, L., Petersson, L., Zelinsky, A.: Driver assistance systems based on vision in and out of vehicles. In: IEEE Proceedings of Intelligent Vehicles Symposium, pp. 322-327 (2003)

[2] WHO (2009). Global Status Report on Road Safety Geneva, Switzerland. https://afro.who.int/sites/default/files/201706/vid_global_status_report_en.pdf [PubMed]

[3] SKL Lal and A. Craig, "A critical review of the psychophysiology of driver fatigue", Biological psychology, vol. 55, no. 3, pp. 173-194, 2021.

[4] AF Abate, M Nappi, D Piccio and G. Sabatino, "2D and 3D face recognition: a survey", Pattern recognition letters 2007, vol. 28, pp. 1885-906.

[5] M Kim, D Lee and KY. Kim, "System Architecture for Real-Time Face Detection on Analog Video Camera", International Journal of Distributed Sensor Networks 2015, pp. 11

[6] G. D. Brito Moncayo y and W. L. Lépesz Romero, "Sistema de control del estado de somnolencia en conductores de vehículos", Universidad Técnica de Ambato Ambato, 2016. 
[7] K. Saldaña Altamirano y and R. Rodríguez Urquiaga, "Detección de la somnolencia usando maquinas de soporte vectorial a partir de" in Universidad Nacional De Trujillo, Trujillo, 2014.

[8] Understanding the "eye aspect ratio"

https://www.pyimagesearch.com/2017/04/24/eye-blink-detection-opencv-python-dlib/, 2021

[9] A. Picot, S. Charbonnier, A. Caplier and N.-S. Vu, "Using retina modelling to characterize blinking: Comparison between EOG and video analysis", Mach. Vis. Appl., vol. 23, no. 6, pp. 1195-1208, Nov. 2012.

[10] Y. Zhang and C. Hua, "Driver fatigue recognition based on facial expression analysis using local binary patterns", Optik, vol. 126, no. 23, pp. 4501-4505, Dec. 2015.

[11] Xue Liu, Xiaoyang Tan, Songcan Chen, " Eye Closeness Detection Using Appearance Based Methods," Intelligent Information Processing, volume 385 of IFIP Advances in Information, Communication Technology, pp. 398-408, 2012. 